\title{
Finite vertex-primitive and vertex-biprimitive 2-path-transitive graphs
}

\author{
Cai Heng Li · Hua Zhang
}

Received: 6 February 2011 / Accepted: 16 November 2011 / Published online: 3 December 2011

(C) Springer Science+Business Media, LLC 2011

\begin{abstract}
This paper presents a classification of vertex-primitive and vertex-biprimitive 2-path-transitive graphs which are not 2-arc-transitive. The classification leads to constructions of new examples of half-arc-transitive graphs.
\end{abstract}

Keywords Symmetric graphs $\cdot$ 2-path-transitive graphs $\cdot$ Half-transitive graphs

\section{Introduction}

Let $\Gamma=(V, E)$ be a graph with vertex set $V$ and edge set $E$. Recall that an arc is an ordered pair of adjacent vertices. Each edge $\{\alpha, \beta\}$ corresponds to two $\operatorname{arcs}(\alpha, \beta)$ and $(\beta, \alpha)$. A 2 -arc in $\Gamma$ is a triple $(\alpha, \beta, \gamma)$ of three distinct vertices such that $\beta$ is adjacent to both $\alpha$ and $\gamma$. Identifying the 2 -arcs $(\alpha, \beta, \gamma)$ and $(\gamma, \beta, \alpha)$ gives rise to a 2-path, denoted by $[\alpha, \beta, \gamma]$.

A graph $\Gamma$ is called $G$-arc-transitive, $(G, 2)$-arc-transitive, or $(G, 2)$-pathtransitive if $G \leq$ Aut $\Gamma$ acts transitively on the set of arcs, the set of 2-arcs, or the set of 2-paths, respectively, in which case $\Gamma$ is sometimes simply called a symmetric graph, a 2-arc-transitive graph, or a 2-path-transitive graph.

The study of symmetric graphs forms a significant part of current research efforts in algebraic graph theory. An important subclass of symmetric graphs is the class of

C.H. Li · H. Zhang $(\bowtie)$

School of Mathematics and Statistics, The University of Western Australia, Crawley, WA 6009,

Australia

e-mail: zhanghua@maths.uwa.edu.au

C.H. Li

e-mail: li@maths.uwa.edu.au

H. Zhang

School of mathematics, Yunnan Normal University, Kunming 650091, P.R. China 
2-arc-transitive graphs, the study of which has been a topic of active research for almost half a century, see $[3,8,11,17,18]$ and the references therein. It follows from the definition that a 2-arc-transitive graph is 2-path-transitive, and 2-arc-transitive graphs thus form a subclass of the class of 2-path-transitive graphs. In 1996, Conder and Praeger initiated the study of 2-path-transitive graphs. This study was further developed in [12]. Apart from being a natural generalization of 2-arc-transitive graphs, this broader class of graphs is worth studying for several other reasons.

Recall that a graph $\Gamma=(V, E)$ is called half-arc-transitive (or half-transitive) if Aut $\Gamma$ is transitive on $V$ and $E$, but not on the set of arcs. The class of half-arctransitive graphs has been widely studied in the past twenty years, and a substantial amount of work was devoted to constructing new half-arc-transitive graphs. Our main motivation for studying 2-path-transitive graphs comes from the fact that there is a close relationship between 2-path-transitivity and half-arc-transitivity. It is shown in [12] that the line graphs of 2-path-transitive but not 2-arc-transitive graphs are halfarc-transitive. This link provides a method of constructing half-arc-transitive graphs. Furthermore, it naturally leads to investigating the gap between '2-path-transitive' and '2-arc-transitive' in general. It is therefore useful to study 2-path-transitive graphs which are not 2-arc-transitive.

On the other hand, the concept of 2-path-transitive graphs may also be viewed as a generalization of cubic arc-regular graphs, another class of graphs that has received extensive attention in the literature. Here a graph $\Gamma$ is called arc-regular (or 1-regular) if Aut $\Gamma$ acts regularly on the set of arcs. It is easy to see that arc-regular cubic graphs are precisely cubic graphs that are 2-path-transitive but not 2-arc-transitive. Therefore the line graphs of cubic arc-regular graphs are half-arc-transitive of valency 4. Based on this observation, a study of arc-regular cubic graphs was carried out in [16], which provides a generic method for constructing half-arc-transitive graphs of valency 4 . The process in turn stimulates a further study on arc-regular graphs.

We note that a 2-path-regular graph is not 2-arc-transitive. Here a graph $\Gamma$ is called 2-path-regular if Aut $\Gamma$ acts regularly on the set of 2-paths of $\Gamma$. The concept of 2-arcregular graphs is similarly defined, and 2-arc-regular graphs are studied in $[8,13]$, for example. As a special case of 2-path-transitive graphs, the study of 2-path-regular graphs is clearly an extension of that of 2-arc-regular graphs.

The aim of this paper is to classify vertex-primitive and vertex-biprimitive 2-pathtransitive graphs which are not 2-arc-transitive, and then to apply the classification to construct new examples of half-arc-transitive graphs.

In this paper our conventions for expressing the structure of groups run as follows. If $H$ and $K$ are groups, then $H . K$ denotes any extension of $H$ by $K, H: K$ denotes a split extension of $H$ by $K$, and the symbol $[m]$ denotes an arbitrary group of order $m$. Furthermore, we use the symbols Th, B and M to denote the Thompson simple group, the Baby Monster simple group and the Monster simple group, respectively.

For the vertex-primitive case, the result is given in the following theorem. (A graph $\Gamma=(V, E)$ is called $G$-vertex-primitive if $G \leq \operatorname{Aut} \Gamma$ is primitive on vertex set $V$.)

Theorem 1.1 Let $\Gamma$ be a $G$-vertex-primitive, $(G, 2)$-path-transitive graph of valency $k$. Assume that $\Gamma$ is not $(G, 2)$-arc-transitive. Then $k=p^{e} \equiv 3(\bmod 4)$ with $p$ prime, and one of the following holds: 
(i) $G=\mathbb{Z}_{2}^{d}: G_{\alpha}$, where $G_{\alpha}$ is a 2-homogeneous permutation group of degree $p^{e}$.

(ii) $G=\mathrm{PSL}_{2}\left(p^{e}\right)$, and $\Gamma=\mathrm{K}_{p^{e}+1}$.

(iii) $G=\mathrm{A}_{p}, G_{\alpha}=\mathbb{Z}_{p}: \mathbb{Z}_{(p-1) / 2}$, and $k=p$ with $p \neq 7,11,23$. Furthermore, either Aut $\Gamma=\mathrm{A}_{p}$ and $\Gamma$ is 2-path-regular, or Aut $\Gamma=\mathrm{S}_{p}$ and $\Gamma$ is 2-arc-regular.

(iv) Aut $\Gamma=G$ is a sporadic simple group, $\Gamma$ is 2-path-regular, and $\left(G, G_{\alpha}\right)$ lies in the following table:

\begin{tabular}{llll}
\hline$G$ & Th & B & M \\
\hline$G_{\alpha}$ & $\mathbb{Z}_{31}: \mathbb{Z}_{15}$ & $\mathbb{Z}_{47}: \mathbb{Z}_{23}$ & $\mathbb{Z}_{59}: \mathbb{Z}_{29}$ or $\mathbb{Z}_{71}: \mathbb{Z}_{35}$ \\
\hline
\end{tabular}

A permutation group $G$ on $\Omega$ is said to be biprimitive if $\Omega$ has a $G$-invariant partition $\Omega=\Delta_{1} \cup \Delta_{2}$, such that the setwise stabilizer $G_{\Delta_{i}}$ is primitive on $\Delta_{i}$. A graph $\Gamma=(V, E)$ is called $G$-vertex-biprimitive if $G \leq$ Aut $\Gamma$ is biprimitive on the vertex set $V$.

Theorem 1.2 Let $\Gamma$ be a connected $(G, 2)$-path-transitive graph of valency $k$ which is not $(G, 2)$-arc-transitive. Assume further that $\Gamma$ is $G$-vertex-biprimitive. Then $\Gamma$ is bipartite, $k=p^{e} \equiv 3(\bmod 4)$ with $p$ prime, and one of the following holds:

(1) $\Gamma$ is the standard double cover of a vertex-primitive graph as given in Theorem 1.1 .

(2) $G=\left(\mathbb{Z}_{r}^{d}: G_{\alpha}\right): \mathbb{Z}_{2}$, where $r$ is prime, $d \geq 1$, and $G_{\alpha}$ is a 2-homogeneous permutation group of degree $p^{e}$ and is an irreducible subgroup of $\mathrm{GL}_{d}(r)$.

(3) $\Gamma=\mathrm{K}_{p^{e}, p^{e}}$, and $G=\left(\mathbb{Z}_{p}^{e} \times \mathbb{Z}_{p}^{e}\right): G_{\alpha \beta}: \mathbb{Z}_{2}$, where $G_{\alpha \beta} \leq \Gamma \mathrm{L}_{1}\left(p^{e}\right) \times \Gamma \mathrm{L}_{1}\left(p^{e}\right)$.

(4) Aut $\Gamma=G=\mathrm{S}_{p}, G_{\alpha}=\mathbb{Z}_{p}: \mathbb{Z}_{(p-1) / 2}$, where $p \equiv 3(\bmod 4)$ is prime, $p \neq 7,11$, 23 , and $\Gamma$ is 2-path-regular.

(5) $G=\mathrm{PGL}_{3}(4) . \mathbb{Z}_{2}, G_{\alpha}=\left(\mathbb{Z}_{7}: \mathbb{Z}_{3}\right) \times \mathbb{Z}_{3}, k=7$, Aut $\Gamma=\operatorname{Aut}\left(\mathrm{PSL}_{3}(4)\right)=G .2$, and $\Gamma$ is 2-arc-transitive.

(6) Aut $\Gamma=G=\mathrm{P} \Gamma \mathrm{U}_{3}(5), G_{\alpha}=\left(\mathbb{Z}_{7}: \mathbb{Z}_{3}\right) \times \mathbb{Z}_{3}$, and $k=7$.

Applying the classification result of Theorems 1.1 and 1.2, we construct half-arctransitive graphs in Theorem 1.3. Some of these graphs are new.

Theorem 1.3 For each group $G$ and a subgroup $H<G$ in Table 1 , there exists a half-arc-transitive graph $\Sigma$ of valency $m$ such that Aut $\Sigma=G$ and $H$ is the stabilizer in $G$ of some vertex of $\Sigma$.

This paper is organised as follows: examples of the graphs given in these three theorems are constructed in Sect. 2. A reduction is given in Sect. 3 for the proofs of Theorems 1.1 and 1.2, and finally Theorems 1.1 and 1.2 are proved in Sect. 4.

\section{Examples}

In this section, we construct and study examples of 2-path-transitive graphs given in Theorems 1.1 and 1.2. Recall that a permutation group $G$ on $\Omega$ is called 2-transitive 
Table 1 Pairs $(G, H)$

associated with half-transitivegraphs

\begin{tabular}{lll}
\hline$G$ & $H$ & $m$ \\
\hline $\mathrm{P} \Gamma \mathrm{U}_{3}(5)$ & $\mathbb{Z}_{3}^{2}: \mathbb{Z}_{2}$ & 12 \\
$\mathrm{Th}$ & $\mathbb{Z}_{15}: \mathbb{Z}_{2}$ & 60 \\
$\mathrm{~B}$ & $\mathbb{Z}_{23}: \mathbb{Z}_{2}$ & 92 \\
$\mathrm{M}$ & $\mathbb{Z}_{29}: \mathbb{Z}_{2}$ & 116 \\
& $\mathbb{Z}_{35}: \mathbb{Z}_{2}$ & 140 \\
$\mathrm{~A}_{p}$ & $\mathrm{D}_{p-1}$ & $2(p-1)$, where $p \equiv 3(\bmod 4)$ is prime, and \\
& & $p \neq 7,11$ or 23 \\
$\mathrm{~S}_{p}$ & $\mathrm{D}_{p-1}$ & $2(p-1), p$ is as above \\
\hline
\end{tabular}

or 2-homogeneous if $G$ induces a transitive action on the set of ordered pairs or on the set of 2-subsets of $\Omega$, respectively.

Let $G$ be a finite group, and let $H$ be a core-free subgroup of $G$. Denote by [ $G: H]$ the set of right cosets of $H$ in $G$, namely $[G: H]=\{H x \mid x \in G\}$. For an element $g \in G$ with $g^{2} \in H$, the Sabidussi coset graph of $G$ with respect to $H$ and $g$ is the graph with vertex set $[G: H]$, such that $H x$ and $H y$ are adjacent if and only if $y x^{-1} \in$ $H g H$. This coset graph is $G$-arc-transitive and is denoted by $\operatorname{Cos}(G, H, H g H)$.

Assume that $\Gamma=\operatorname{Cos}(G, H, H g H)$ is a $(G, 2)$-path-transitive graph which is not $(G, 2)$-arc-transitive. Denote by $\alpha, \beta$ the vertices corresponding to $H$ and $H g$, respectively. Then $(\alpha, \beta)^{g}=(\beta, \alpha)$, and $g^{2} \in G_{\alpha}$. By [4, Theorem 2], $G_{\alpha}=H$ is 2homogeneous but not 2-transitive on the neighborhood of $\alpha$, and furthermore, $\left|G_{\alpha}\right|$ is odd. Thus $g^{2}=1$, that is, $g$ is an involution. Also $\Gamma$ is connected if and only if $\langle H, g\rangle=G$.

To construct 2-path-transitive graphs which are not 2-arc-transitive, we need the following result, the proof of which is straightforward.

Lemma 2.1 Let $G$ be a finite group with a core-free subgroup $H$ and an involution $g$. Assume further that $\langle H, g\rangle=G$, and the coset action of $H$ on $\left[H: H \cap H^{g}\right]$ is 2homogeneous but not 2-transitive. Then the coset graph $\operatorname{Cos}(G, H, H g H)$ is $(G, 2)$ path-transitive but not $(G, 2)$-arc-transitive.

Example 2.2 Let $\Gamma=\mathrm{K}_{q+1}$, where $q=p^{e} \equiv 3(\bmod 4)$, with $p$ prime. Then Aut $(\Gamma)=\mathrm{S}_{q+1}$ contains a subgroup $G=\operatorname{PSL}_{2}(q)$. For a vertex $\alpha$ of $\Gamma$, we have $G_{\alpha}=\mathbb{Z}_{p}^{e}: \mathbb{Z}_{\left(p^{e}-1\right) / 2}$, which is a maximal subgroup of $G$. Thus by Lemma 2.1, the graph $\Gamma$ is $G$-vertex-primitive, and $(G, 2)$-path-transitive but not $(G, 2)$-arctransitive.

Example 2.3 Let $\Gamma=\mathrm{K}_{p^{e}, p^{e}}$, where $p^{e} \equiv 3(\bmod 4)$, be the complete bipartite graph of order $2 p^{e}$. Then $\operatorname{Aut}(\Gamma)=\mathrm{S}_{p^{e}} 2 \mathbb{Z}_{2}$. Let $G=\left(\left(\mathbb{Z}_{p}^{e} \times \mathbb{Z}_{p}^{e}\right): H\right): \mathbb{Z}_{2}<\operatorname{Aut}(\Gamma)$, where $\mathbb{Z}_{\left(p^{e}-1\right) / 2} \leq H \leq \Gamma \mathrm{L}_{1}\left(p^{e}\right)$, and $|H|$ is odd. Then the graph $\Gamma$ is $G$-vertexbiprimitive, $(G, 2)$-path-transitive but not $(G, 2)$-arc-transitive. The index two subgroup $G^{+}$of $G$ fixes both parts of $\Gamma$, and is primitive of affine type.

Next, we study a family of 2-path-transitive graphs associated with alternating groups. 
Example 2.4 Let $G=\mathrm{S}_{p}$, and $T=\mathrm{A}_{p}$, both acting naturally on the set $\{1,2, \ldots, p\}$, where $p$ is prime, such that $p \equiv 3(\bmod 4)$, with $p \neq 7,11,23$. Let $H$ be a subgroup of $T$ isomorphic to $K: L$, where $K=\mathbb{Z}_{p}$ and $L=\mathbb{Z}_{(p-1) / 2}$. Then $H$ is a maximal subgroup of $T$. Since subgroups which are isomorphic to $H$ are conjugate in $T$, it follows that $L$ is conjugate to $\left\langle t^{2}\right\rangle$, where $t$ is a $(p-1)$-cycle. Let $g \in \mathbf{N}_{T}(L) \backslash L$, and $f \in \mathbf{N}_{G}(L) \backslash \mathrm{A}_{p}$ be involutions. Define

$$
\Gamma=\operatorname{Cos}(T, H, H g H) \quad \text { and } \quad \Sigma=\operatorname{Cos}(G, H, H f H) .
$$

For example, we may choose $t=(1,2, \ldots, p-1)$. Then

$$
t^{2}=(1,3, \ldots, p-2)(2,4, \ldots, p-1),
$$

and $g$ and $f$ can be defined as follows:

$$
\begin{aligned}
g= & (3, p-2)(5, p-4) \ldots\left(\frac{p-1}{2}, \frac{p+3}{2}\right)(4, p-1)(6, p-3) \ldots \\
& \left(\frac{p+1}{2}, \frac{p+5}{2}\right) \in \mathrm{A}_{q}, \\
f= & (1,2)(3,4) \ldots(p-2, p-1) \in \mathrm{S}_{q} \backslash \mathrm{A}_{q} .
\end{aligned}
$$

The graph $\Gamma$ is connected, $(T, 2)$-path-transitive but not $(T, 2)$-arc-transitive, and Aut $\Gamma=T$ or $G$; see [12] for details. Further, since $H$ is a maximal subgroup of $T$, $\Gamma$ is also $T$-vertex-primitive.

For the graph $\Sigma$, we have the following conclusion.

Lemma 2.5 Let $\Sigma$ be the graph constructed in Example 2.4. Then $\Sigma$ is connected, vertex-biprimitive, and 2-path-regular; furthermore, Aut $\Sigma=\mathrm{S}_{p}$.

Proof By definition, $f \in G \backslash T$ normalizes $L$. Since $H<T$, and $G / T \cong \mathbb{Z}_{2}, T$ has exactly two orbits on $[G: H]$, say $\Delta_{1}$ and $\Delta_{2}$, such that $G^{+}:=G_{\Delta_{i}}=T$. Moreover, the setwise stabilizer $G^{+}$is primitive on $\Delta_{i}$, and $\Sigma$ is a bipartite graph with parts $\Delta_{1}$ and $\Delta_{2}$. Since $f$ does not normalize $H$, it follows that $\Sigma$ is connected. Since $H \cap H^{f}=L$, and the action of $H$ on $\left[H: H \cap H^{f}\right]$ is 2-homogeneous but not 2-transitive, we conclude that $\Sigma$ is a $(G, 2)$-path-transitive graph of valency $p$, which is not $(G, 2)$-arc-transitive. Further, $\Gamma$ is $G$-vertex-biprimitive.

Suppose that $G^{+}$is not normal in Aut $\Sigma$. Then there exists a group $X$ such that $T<X \leq$ Aut $\Sigma$, and $\mathbf{N}_{\text {Aut } \Sigma}(T)$ is a maximal subgroup of $X$. Since $T$ is primitive on $\Delta_{i}$, so is $X$. Since $\left|\Delta_{i}\right|=|T: H|=(p-2)$ !, by the O'Nan-Scott Theorem, we conclude that either $X$ is almost simple or $X \triangleright \mathrm{A}_{p-2} \times \mathrm{A}_{p-2}$. Moreover, because the order $|X|$ is divisible by $p$, it follows that $X$ is almost simple. Thus we have $X=\mathbf{N}_{\text {Aut } \Gamma}(T) X_{\alpha}$ is a maximal factorisation of the almost simple group $X$. Therefore the triple $\left(X, \mathbf{N}_{\mathrm{Aut} \Gamma}(T), X_{\alpha}\right)$ should lie in the classification of [15]. However, an inspection shows that there is no such a triple, which is a contradiction. Thus $G^{+}=T$ is normal in Aut $\Sigma$. Since $T$ is primitive on $\Delta_{i}$, the centralizer of $T$ in Aut $\Sigma$ is trivial, so Aut $\Sigma \leq \operatorname{Aut}(T)=\mathrm{S}_{p}$. Since Aut $\Sigma \geq G=\mathrm{S}_{p}$, we have Aut $\Sigma=\mathrm{S}_{p}$. 
Let $\Gamma=\operatorname{Cos}(G, H, H g H)$, and let $\operatorname{Aut}(G, H)=\left\{\sigma \in \operatorname{Aut}(G) \mid H^{\sigma}=H\right\}$. The following conclusion is well-known, and the proof is easy.

Lemma 2.6 Suppose that $\sigma \in \operatorname{Aut}(G, H)$. Then $\Gamma=\operatorname{Cos}(G, H, H g H)$ is isomorphic to $\Sigma=\operatorname{Cos}\left(G, H, \mathrm{Hg}^{\sigma} \mathrm{H}\right)$. Moreover, $\sigma$ induces an automorphism of $\Gamma$ if and only if $\mathrm{HgH}=\mathrm{Hg}^{\sigma} \mathrm{H}$.

Let $\Gamma$ be the graph and $t$ be a $(p-1)$-cycle defined in Example 2.4. Let $M=\langle t\rangle$. We point out that the choice of $g$ for defining $\Gamma$ is not unique. Indeed we can even choose $g$ such that $g \in \mathbf{N}_{T}(L) \backslash \mathbf{N}_{T}(M)$. This is because $\mathbf{N}_{T}(L)$ is an index two subgroup of $\mathbf{N}_{G}(L)$, and further, as $\mathbf{N}_{T}(M) \leq \mathbf{N}_{T}(L)$, and $(p-1) / 2$ is odd, we have $\mathbf{N}_{G}(M)=M: \operatorname{Aut}(M) \cong \mathbb{Z}_{p-1}: \operatorname{Aut}\left(\mathbb{Z}_{p-1}\right)=\mathbb{Z}_{p-1}: \operatorname{Aut}\left(\mathbb{Z}_{(p-1) / 2}\right)$. Since $\mathbf{N}_{T}(L) \geq \mathbb{Z}_{p-1}:\left(\operatorname{Aut}\left(\mathbb{Z}_{(p-1) / 2}\right) \times \operatorname{Aut}\left(\mathbb{Z}_{(p-1) / 2}\right)\right)$, we conclude that $\mathbf{N}_{T}(M)<\mathbf{N}_{T}(L)$, and a Sylow-2 subgroup of $\mathbf{N}_{T}(M)$ is properly contained in a Sylow-2 subgroup of $\mathbf{N}_{T}(L)$. Therefore there exists a 2-element $g$ such that $g \in \mathbf{N}_{T}(L) \backslash \mathbf{N}_{T}(M)$. Actually the full automorphism group of $\Gamma$ depends on the choice of $g$.

The case Aut $\Gamma=\mathrm{S}_{p}$ does occur.

Lemma 2.7 If $g \in \mathbf{N}_{T}(M) \backslash M$, then Aut $\Gamma=\mathrm{S}_{p}$ and $\Gamma$ is 2-arc-regular.

Proof Let $H_{1}=K: M$, and let $V_{1}=\left[G: H_{1}\right]$. Then $H$ is an index 2 subgroup of $H_{1}$. Now the graph $\Gamma_{1}=\operatorname{Cos}\left(G, H_{1}, H_{1} g H_{1}\right)$ is 2-arc regular, with full automorphism group $\mathrm{S}_{p}$ (see [8, Lemma 4.2]). Further, since $T$ is transitive on $V_{1}$, for any $H_{1} x \in V_{1}$ we may choose $x \in T$. Then it is straightforward to show that the map $\psi: V_{1} \mapsto V$ defined by $\psi\left(H_{1} x\right)=H x$ is a graph isomorphism between $\Gamma_{1}$ and $\Gamma$. It follows that Aut $\Gamma=\mathrm{S}_{p}$, and $\Gamma$ is 2-arc-regular.

The case Aut $\Gamma=\mathrm{A}_{p}$ occurs too.

Lemma 2.8 If $g \in \mathbf{N}_{T}(L) \backslash \mathbf{N}_{T}(M)$, then Aut $\Gamma=\mathrm{A}_{p}$ and $\Gamma$ is 2-path-regular.

Proof As indicated in Example 2.4, Aut $\Gamma=\mathrm{A}_{p}$ or $\mathrm{S}_{p}$. Suppose that Aut $\Gamma=\mathrm{S}_{p}$. Write $M=L \times\langle\sigma\rangle$, where $o(\sigma)=2$. Then $\left\langle\mathrm{A}_{p}, \sigma\right\rangle=\mathrm{S}_{p}$ and $H^{\sigma}=H$. Thus by Lemma 2.6, we have $H g H=H g^{\sigma} H$. Since $g \notin \mathbf{N}_{T}(M)$, we obtain $g^{\sigma} \neq g$. Since $g^{\sigma} \in H g H$ and $g \in \mathbf{N}_{T}(L)$, there exist $a, b \in K$ and $u \in L$ such that $g^{\sigma}=a g b u$. As $o\left(g^{\sigma}\right)=2$, we have $(a g b u)^{2}=1$, so $(b u a)^{g}=(b u a)^{-1}$. Since bua $\in H$, we obtain bua $\in H \cap H^{g}=L$. As $u \in \mathbf{N}_{T}(K)$, we have $u^{-1} b u a=b^{u} a \in K \cap L$, that is, $b^{u} a=1$. Thus $g^{\sigma}=(g u)^{a^{-1}}$. Since $g^{\sigma}$ normalizes $L$, we have $\left(L^{a}\right)^{g u}=L^{a}$. Notice that $L^{a} \in H$, so $L^{a} \leq H \cap H^{g u}=L$, and therefore $L^{a}=L$. If $a \neq 1$, then the order of $a$ is $p$, which means that $K$ normalizes $L$, which is a contradiction. Thus $a=b=1$ and $g^{\sigma}=g u$. Since both $g$ and $\sigma$ are involutions, we obtain $\sigma^{g}=u \sigma$, that is, $g$ normalizes $M$, which is again a contradiction. Thus Aut $\Gamma=\mathrm{A}_{p}$, as claimed.

The graphs in the next example arise from three sporadic simple groups: the Thompson group Th, the Baby Monster B, and the Monster M. 
Example 2.9 Let $G=\mathrm{Th}$, B or M. Then by the Atlas [5], the group $G$ contains a maximal subgroup $H$ such that $H \cong \mathbb{Z}_{p}: \mathbb{Z}_{(p-1) / 2}$, where $p=31$ for $G=\mathrm{Th}, p=$ 47 for $G=\mathrm{B}$, and $p=59$ or 71 for $G=\mathrm{M}$. Let $L$ be a subgroup of $H$ which is isomorphic to $\mathbb{Z}_{(p-1) / 2}$. Then in each case, $\mathbf{N}_{G}(L)$ is of even order. This is true because the Atlas [5] shows that for $G=\mathrm{Th},\left|\mathbf{C}_{G}(L)\right|=30$; for $G=\mathrm{B},\left|\mathbf{C}_{G}(L)\right|=$ 46; for $G=\mathrm{M}$ with $p=59, \mathbf{N}_{G}(L)=\left(\mathbb{Z}_{29}: \mathbb{Z}_{14} \times 3\right) .2$; and for $G=\mathrm{M}$ with $p=71$, $\left|\mathbf{C}_{G}(L)\right|=70$ or 2100 .

Let $g$ be an involution in $\mathbf{N}_{G}(L)$, and let $\Gamma=\operatorname{Cos}(G, H, H g H)$. Then $H \cap H^{g}=$ $L$, hence $\Gamma$ has valency $|H: L|=p$. Since $H$ is a maximal subgroup of $G$, we have $\langle H, g\rangle=G$, thus $\Gamma$ is connected. Further, since the action of $H$ on $[H: L]$ is 2-homogeneous and is not 2-transitive, we conclude that $\Gamma$ is $G$-vertex-primitive, $(G, 2)$-path-transitive but not $(G, 2)$-arc-transitive.

Moreover, for the graphs that we just constructed in Example 2.9, the following conclusion is true:

Lemma 2.10 If $\Gamma$ is a graph in Example 2.9, then Aut $\Gamma=G$, and $\Gamma$ is 2-pathregular.

Proof Suppose that $G$ is not normal in Aut $\Gamma$. Then there exists a group $X$ such that $G<X \leq$ Aut $\Gamma$ and $\mathbf{N}_{\text {Aut } \Gamma}(G)$ is a maximal subgroup of $X$. Since $G$ is primitive on $V \Gamma$, so is $X$. Also because $\Gamma$ is $(G, 2)$-path-transitive, we have either $\Gamma$ is $(X, 2)$ arc-transitive, or $\Gamma$ is $(X, 2)$-path-transitive but not $(X, 2)$-arc-transitive. Thus the primitive type of $X$ is affine, almost simple, product action or twisted wreath product. Notice that $|V \Gamma|=|G: H|$ is not a power of an integer, and $|G: H|$ is exactly divisible by 19 , we conclude that $X$ is almost simple. Thus $X=\mathbf{N}_{\text {Aut } \Gamma}(G) X_{\alpha}$ is a maximal factorisation, and hence the triple $\left(X, \mathbf{N}_{\mathrm{Aut} \Gamma}(G), X_{\alpha}\right)$ lies in the classification given in [15]. However, an inspection of the classification shows that there is no such a triple, which is a contradiction. Therefore $G$ is normal in Aut $\Gamma$. Since $G$ is primitive on $V$, the centralizer of $G$ in $\operatorname{Aut} \Gamma$ is trivial. As Out $(G)=1$, we conclude that Aut $\Gamma=$ $G$, and $\Gamma$ is 2-path-regular.

The following two examples arise from classical groups of Lie type.

Example 2.11 Let $T=\mathrm{PGL}_{3}(4)$. Then by the Atlas [5], $T$ contains a maximal subgroup $H$ which is isomorphic to $\left(\mathbb{Z}_{7}: \mathbb{Z}_{3}\right) \times \mathbb{Z}_{3}$. Let $G=\operatorname{PGL}_{3}(4) .\langle\tau\rangle$, where $\tau$ is a field automorphism or the graph automorphism of $\operatorname{PSL}_{3}(4)$, of order 2 . Write $H=K: L=(\langle x\rangle:\langle y\rangle) \times\langle z\rangle$, where $K=\langle x\rangle, L=\langle y\rangle \times\langle z\rangle, o(x)=7$, $o(y)=o(z)=3$, and $z$ is a diagonal automorphism of $\mathrm{PSL}_{3}(4)$. Since subgroups of $G$ which are isomorphic to $H$ are conjugate, we may assume that $x, y \in \mathrm{PGL}_{3}(2)$. Then $z x=x z$ and $z y=y z$. By the Atlas [5], $\mathbf{N}_{G}(K)=(7: 3 \times 3)$.2. Since $x y \neq y x$, we have $\left|\mathbf{C}_{G}(K)\right|=21$ or 42 . If $\left|\mathbf{C}_{G}(K)\right|=42$, then $\mathbf{N}_{G}(K)$ contains only three involutions. Assume that $\left|\mathbf{C}_{G}(K)\right|=21$. Then $\mathbf{N}_{G}(K) / \mathbf{C}_{G}(K)=\mathbb{Z}_{6}$. Thus there are at most 21 involutions in $\mathbf{N}_{G}(K)$. Suppose that $a \in \mathbf{N}_{G}(K)$ is an involution such that $a \in \mathbf{N}_{G}(L)$. We claim that none of the involutions $a^{x^{k}}$, where $1 \leq k \leq 6$, is contained in $\mathbf{N}_{G}(L)$. Assume that $a^{x^{k}} \in \mathbf{N}_{G}(L)$. Then $a a^{x^{k}} \in \mathbf{N}_{G}(L)$. By definition, $\langle x, a\rangle$ is a 
dihedral group of order 14 , and so $a a^{x^{k}}$ has order 7. Since $\left|\mathbf{N}_{T}(L)\right|=27$, we have $\left|\mathbf{N}_{G}(L)\right|$ is 27 or 54 . Since $\left|\mathbf{N}_{G}(L)\right|$ is even, we have $\left|\mathbf{N}_{G}(L)\right|=54$. But $7 \nmid\left|\mathbf{N}_{G}(L)\right|$, a contradiction. It follows that among the involutions of $\mathbf{N}_{G}(K)$, at most three are contained in $\mathbf{N}_{G}(L)$. Thus there exists an involution $g \in \mathbf{N}_{G}(L) \backslash \mathbf{N}_{G}(K)$, and the graph $\Gamma=\operatorname{Cos}(G, H, H g H)$ is $G$-vertex-biprimitive, $(G, 2)$-path-transitive but not $(G, 2)$-arc-transitive, of valency 7 .

Example 2.12 Let $T=\mathrm{PGU}_{3}(5)$. Then by the Atlas [5], $T$ contains a maximal subgroup $H$ which is isomorphic to $\left(\mathbb{Z}_{7}: \mathbb{Z}_{3}\right) \times \mathbb{Z}_{3}$. Let $G=\mathrm{P} \Gamma \mathrm{U}_{3}(5)$. As in Example 2.11, write $H=K: L$. Then by GAP [9], $\mathbf{N}_{G}(L)$ contains nine involutions, three of which are contained in $\mathbf{N}_{G}(K)$. Thus for any 2-element $g \in \mathbf{N}_{G}(L) \backslash \mathbf{N}_{G}(K)$, the graph $\Gamma=\operatorname{Cos}(G, H, H g H)$ is $G$-vertex-biprimitive, $(G, 2)$-path-transitive but not $(G, 2)$-arc-transitive, of valency 7 .

For the graphs in Examples 2.11 and 2.12, the full automorphism groups of the graphs are determined in the next two lemmas.

Lemma 2.13 If $\Gamma$ is the graph in Example 2.11, then Aut $\Gamma=\operatorname{PSL}_{3}(4) .\left(2 \times \mathrm{S}_{3}\right)$ and $\Gamma$ is 2-arc-transitive.

Proof By definition, $G=\mathrm{PGL}_{3}(4) .\langle\tau\rangle$, where $\tau$ is a field automorphism or the graph automorphism of $\mathrm{PSL}_{3}(4)$, of order 2. Assume first that $\tau$ is the graph automorphism of $\mathrm{PSL}_{3}(4)$ (that is, $\tau$ is the transpose inverse map). Now $\Gamma=\operatorname{Cos}\left(G, G_{\alpha}, G_{\alpha} g G_{\alpha}\right)$, with $G_{\alpha}=7: 3 \times 3$. Let $T=\mathrm{PGL}_{3}(4)$ and consider $G_{\alpha}$ as a semi-direct product $K: L=(\langle x\rangle:\langle y\rangle) \times\langle z\rangle$, where $K=\langle x\rangle, L=\langle y\rangle \times\langle z\rangle, o(x)=7, o(y)=o(z)=3$, and $z$ is a diagonal automorphism of $\operatorname{PSL}_{3}(4)$. Then the 2-element $g$ lies in $\mathbf{N}_{G}(L) \backslash \mathbf{N}_{G}(K)$.

Let $\sigma$ be a field automorphism of $\mathrm{PSL}_{3}(4)$. We claim that $g^{\sigma} \in H g H$.

As observed in Example 2.11, the normaliser $\mathbf{N}_{T}(L)$ is a Sylow 3-subgroup of $G$, with $\left|\mathbf{N}_{T}(L)\right|=27$ and $\left|\mathbf{N}_{G}(L)\right|=54$. Thus we may write $\mathbf{N}_{T}(L)=(\langle y\rangle \times\langle z\rangle):\langle t\rangle$, where $o(t)=3$. Then $g$ can be written as $g=g_{1} \tau$, where $g_{1}=y^{k_{1}} z^{k_{2}} t^{k_{3}} \in T$, with $k_{i}= \pm 1$. As both $g$ and $\tau$ normalize $L$, so does $g_{1}$. Since $\tau$ normalizes $H$, we have $H g H=H g_{1} \tau H=H g_{1} H \tau$. Because $\sigma \tau=\tau \sigma$, we have $g^{\sigma}=g_{1}^{\sigma} \tau$, and hence $g^{\sigma} \in$ $H g H=H g_{1} H \tau$ if and only if $g_{1}^{\sigma} \in H g_{1} H$. By the Atlas [5], $\sigma$ fixes the Sylow 3subgroup $\mathbf{N}_{T}(L)$, and thus $g_{1}^{\sigma} \in \mathbf{N}_{T}(L)$. Assume that $g_{1}^{\sigma}=y^{l_{1}} z^{l_{2}} t^{l_{3}}=y^{l_{1}} z^{l_{2}} t^{l_{3}-k_{3}} t^{k_{3}}$. Since $t$ normalizes $\langle y\rangle \times\langle z\rangle$, it follows that $g_{1}^{\sigma} \in H g_{1} H$. Thus $\sigma$ induces an automorphism of $\Gamma$. If we interchange the roles of $\tau$ and $\sigma$ by assuming that $\tau$ is a field automorphism of $\mathrm{PSL}_{3}(4)$, and $\sigma$ is the graph automorphism, then analogously we have $\sigma \in$ Aut $\Gamma$. Hence in both cases, we have Aut $\Gamma \geq \operatorname{PGL}_{3}(4) .(\langle\tau\rangle \times\langle\sigma\rangle) \cong$ $\mathrm{PSL}_{3}(4) .\left(2 \times \mathrm{S}_{3}\right)$. We will show that equality holds. Let $A=\mathrm{PSL}_{3}(4) .\left(2 \times \mathrm{S}_{3}\right)$.

Suppose, to the contrary, that $X_{0}:=$ Aut $\Gamma>A$. Then there exists a subgroup $X$ of $X_{0}$ such that $A<X \leq X_{0}$ and $A$ is maximal in $X$. We have two possibilities: $X$ is primitive or biprimitive on $V$.

Assume that $X$ is primitive on $V$. Then $\Gamma$ is $(X, 2)$-path-transitive. Accordingly, the primitive type of $X$ is either affine, almost simple, product action, or twisted wreath product. Assume first that $X$ is of affine type. Then for any $\alpha \in V, X_{a}$ is 
faithful on $\Gamma(\alpha)$. On the other hand, $\mathrm{A}_{\alpha} \leq X_{\alpha}$, and $\mathrm{A}_{\alpha}$ acts unfaithfully on $\Gamma(\alpha)$, we obtain a contradiction. Since $|V|=1920$, we know that the primitive type of $X$ is neither product action nor twisted wreath product. Thus $X$ is almost simple, and $X=A X_{\alpha}$ is a maximal factorisation. Hence the triple $\left(X, A, X_{\alpha}\right)$ lies in the classification given in [15]. Notice that $|V|=\left|X: X_{\alpha}\right|=1920$. An inspection of the classification shows that there is no such a triple, which is a contradiction.

Assume that $X$ is biprimitive on $V$. Then the two invariant blocks of $X$ are the same as that of $G$, which we suppose to be $\Delta$ and $\Delta^{\prime}$. Then $X$ induces a primitive action on both $\Delta$ and $\Delta^{\prime}$. Since $|V|=1920$, the primitive type of $X$ is not affine, product action, or twisted wreath product. Thus $X_{\Delta}$ is almost simple, and once again we obtain a maximal factorisation $X_{\Delta}=\left(A \cap X_{\Delta}\right)\left(X_{\Delta}\right)_{\alpha}$, where $A \cap X_{\Delta}=\mathrm{PGL}_{3}$ (4).2. An inspection of [15] shows that there is no such a factorisation, a contradiction.

From the above discussion, we come to the conclusion that Aut $\Gamma=\mathrm{PSL}_{3}(4) .(2 \times$ $\mathrm{S}_{3}$ ), and $\Gamma$ is 2 -arc-transitive, as claimed.

Lemma 2.14 Let $\Gamma$ be the graph in Example 2.12. Then Aut $\Gamma=G$, and $\Gamma$ is 2path-transitive but not 2-arc-transitive.

Proof Since Aut $\Gamma \geq G$, we only need to show that equality holds. Suppose, on the contrary, that $X_{0}:=$ Aut $\Gamma>G$. Then there exists a subgroup $X$ of $X_{0}$ such that $G<X \leq X_{0}$, and $G$ is maximal in $X$. In this case we have $|V|=12000$, and the argument in Lemma 2.13 also applies to the current case. Thus Aut $\Gamma=G$.

\section{A reduction}

For a graph $\Gamma=(V, E)$ and a vertex $\alpha$, denote by $\Gamma(\alpha)$ the neighborhood of $\alpha$, that is, the set of vertices to which $\alpha$ is adjacent. For a group $G \leq \operatorname{Aut} \Gamma$, the stabilizer $G_{\alpha}$ induces a natural action on $\Gamma(\alpha)$. As usual, the permutation group induced by $G_{\alpha}$ on $\Gamma(\alpha)$ is denoted by $G_{\alpha}^{\Gamma(\alpha)}$, and the kernel of $G_{\alpha}$ acting on $\Gamma(\alpha)$ is denoted by $G_{\alpha}^{[1]}$. Then $G_{\alpha}^{\Gamma(\alpha)} \cong G_{\alpha} / G_{\alpha}^{[1]}$. Let $\Gamma$ be $(G, 2)$-path-transitive. Then by the well-known Thompson-Wielandt Theorem, for an $\operatorname{arc}(\alpha, \beta)$ of $\Gamma$, the subgroup $G_{\alpha \beta}^{[1]}=G_{\alpha}^{[1]} \cap$ $G_{\beta}^{[1]}$ is a $p$-group with $p$ prime. Moreover, it follows from a result of Weiss (see [18]) that $G_{\alpha \beta}^{[1]}=1$. A characterisation of the vertex stabilizer for a 2-path-transitive graph is then easily obtained in [12], restated below. Recall that for a group $X$ and a prime $p, \mathbf{O}_{p}(X)$ is the largest normal $p$-subgroup of $X$.

Theorem 3.1 (see [12, Theorem 1.1]) Let $\Gamma$ be a connected $(G, 2)$-path-transitive graph which is not $(G, 2)$-arc-transitive. Then $\Gamma$ has valency $p^{e}$, where $p^{e} \equiv$ $3(\bmod 4)$ with $p$ prime, and for each edge $\{\alpha, \beta\}, G_{\alpha}^{\Gamma(\alpha)} \leq \mathrm{A} \Gamma \mathrm{L}_{1}\left(p^{e}\right)$ is 2homogeneous, and the following hold:

(a) $G_{\beta}^{[1]} \cong\left(G_{\beta}^{[1]}\right)^{\Gamma(\alpha)} \triangleleft G_{\alpha \beta}^{\Gamma(\alpha)} \leq \mathbb{Z}_{\left(p^{e}-1\right) / 2}: \mathbb{Z}_{e}<\Gamma \mathrm{L}_{1}\left(p^{e}\right)$, and $G_{\alpha \beta}=\left(G_{\alpha}^{[1]} \times\right.$ $\left.G_{\beta}^{[1]}\right) . O$, where $O \cong G_{\alpha \beta}^{\Gamma(\alpha)} /\left(G_{\beta}^{[1]}\right)^{\Gamma(\alpha)}$.

(b) $\mathbf{O}_{p}\left(G_{\alpha}\right) \cong \mathbb{Z}_{p}^{e}$ is regular on $\Gamma(\alpha)$, and $G_{\alpha}=\mathbf{O}_{p}\left(G_{\alpha}\right): G_{\alpha \beta}$, with order $\left|G_{\alpha}\right|$ dividing $p^{e}\left(\frac{\left(p^{e}-1\right) e}{2}\right)^{2}$. 
For positive integers $q$ and $n$, a prime divisor of $q^{n}-1$ is called a primitive prime divisor if it does not divide $q^{i}-1$ for all $i<n$. The following result is due to Zsigmondy.

Lemma 3.2 (Refer to [10, p. 508]) For any positive integers $q$ and n, either

(i) $q^{n}-1$ has a primitive prime divisor, which is at least $n+1$, or

(ii) $(n, q)=(6,2)$ or $\left(2,2^{b}-1\right)$, where $b \geq 2$ is an integer.

For a prime-power $p^{e} \equiv 3(\bmod 4)$, it is easily shown that $p^{e}-1$ has primitive prime divisors. We thus have the following statement.

Corollary 3.3 If $p^{e}$ is the valency of a 2-path-transitive but not 2-arc-transitive graph, then $p^{e}-1$ has a primitive prime divisor which is at least 5.

Let $\Gamma=(V, E)$ be a connected $(G, 2)$-path-transitive graph which is not $(G, 2)$ arc-transitive. Assume further that $G$ is primitive or biprimitive on $V$. Let

$$
G^{+}=\left\langle G_{\alpha} \mid \alpha \in V\right\rangle \text {. }
$$

Then either $G$ is primitive on $V$ and $G^{+}=G$, or $G$ is biprimitive on $V$, and $G^{+}$is of index 2 in $G$ and has exactly two orbits on $V$ which form the bipartition of $V$. In particular, in either case, $G_{\alpha}=G_{\alpha}^{+}$is maximal in $G^{+}$. The next lemma is a reduction for proving Theorems 1.1 and 1.2.

Lemma 3.4 Let $\Gamma=(V, E)$ be a connected $(G, 2)$-path transitive graph which is not $(G, 2)$-arc-transitive. Assume that $G$ acts primitively or biprimitively on $V$. Then either $\Gamma=\mathrm{K}_{p^{e}}$, pe with $p^{e} \equiv 3(\bmod 4)$, or $G^{+}$is a primitive permutation group of affine type or almost simple type.

Proof Let $\Omega$ be an orbit of $G^{+}$on $V$, and let $\alpha \in \Omega$. By Theorem 3.1, the valency $|\Gamma(\alpha)|$ is $p^{e}$ for some prime $p$ such that $p^{e} \equiv 3(\bmod 4)$.

Suppose that $G^{+}$acts unfaithfully on $\Omega$. Then $\Gamma$ is bipartite with two parts $\Omega$ and $\Omega^{g}$, where $g \in G \backslash G^{+}$. Since $G^{+}$is faithful on $V=\Omega \cup \Omega^{g}$, the kernel $G_{(\Omega)}^{+}$acts on $\Omega^{g}$ non-trivially. Since $G_{(\Omega)}^{+} \triangleleft G^{+}$and $G^{+}$is primitive on $\Omega^{g}$, we conclude that $G_{(\Omega)}^{+}$is transitive on $\Omega^{g}$. It follows that $\Gamma$ is a complete bipartite graph $\mathrm{K}_{|\Omega|,\left|\Omega^{g}\right|}$, of valency $|\Omega|$, and hence $|\Omega|=p^{e}$.

Assume instead that $G^{+}$is faithful on $\Omega$. Since $G$ is primitive or biprimitive on $V$, we have $G^{+} \leq \operatorname{Sym}(\Omega)$ is primitive. By Theorem 3.1, the point stabilizer $G_{\alpha}^{+}$is soluble, and hence in the language of the O'Nan-Scott Theorem (see [7]), the action of $G^{+}$on $\Omega$ is of affine, almost simple, or product action type.

Suppose that $G^{+}$is of product action type. Then $G^{+} \leq\left(\tilde{T}_{1} \times \tilde{T}_{2} \times \cdots \times \tilde{T}_{k}\right): \mathrm{S}_{k}$ $\leq \operatorname{Sym}(\Delta) \gtrless \mathrm{S}_{k}$, and $\Omega=\Delta^{k}$, where $k \geq 2, \tilde{T}_{i} \leq \operatorname{Sym}(\Delta)$ is almost simple with socle $\bar{T}_{i}$, and $\tilde{T}_{i}$ is primitive on $\Delta$. The socle, $N=\operatorname{soc}\left(G^{+}\right)=T_{1} \times \cdots \times T_{k} \cong T^{k}$, where $T_{i} \cong T$, is a minimal normal subgroup of $G^{+}$. Since $G^{+}=N G_{\alpha}^{+}$, we know that $G_{\alpha}^{+}$ induces a transitive action on $\left\{T_{1}, T_{2}, \ldots, T_{k}\right\}$ by conjugation. Since $G_{\alpha}^{+}$is of odd order, it follows that $k$ is odd and so $k \geq 3$. 
By Theorem 3.1, the subgroup $\mathbf{O}_{p}\left(G_{\alpha}\right) \cong \mathbb{Z}_{p}^{e}$ is regular on $\Gamma(\alpha)$ and is a minimal normal subgroup of $G_{\alpha}$. Thus either $\mathbf{O}_{p}\left(G_{\alpha}\right) \leq N_{\alpha}$, or $\mathbf{O}_{p}\left(G_{\alpha}\right) \cap N_{\alpha}=1$. Further, since $N_{\alpha}$ is transitive on $\Gamma(\alpha)$, the order $\left|N_{\alpha}\right|$ is divisible by $p^{e}$. If $\mathbf{O}_{p}\left(G_{\alpha}\right) \cap N_{\alpha}=$ 1 , then $\left|G_{\alpha}\right|$ is divisible by $\left(p^{e}\right)^{2}$, which is not possible by Theorem 3.1. Thus $\mathbf{O}_{p}\left(G_{\alpha}\right) \leq N_{\alpha}$, and $N_{\alpha}=\mathbf{O}_{p}\left(G_{\alpha}\right): N_{\alpha \beta} \unlhd G_{\alpha}$.

Note that $\mathbb{Z}_{p}^{e}: N_{\alpha \beta}=\mathbf{O}_{p}\left(G_{\alpha}\right): N_{\alpha \beta}=N_{\alpha}=\prod T_{i \delta_{i}}$, where $\delta_{i} \in \Delta$. Suppose that $N_{\alpha \beta}=1$. Then $T_{i \delta_{i}}=\mathbb{Z}_{p}^{m}$ (for some $m$ dividing $e$ ) is a maximal subgroup of the almost simple group $\tilde{T}_{i}$, which is impossible. Hence $N_{\alpha \beta} \neq 1$. Since $\mathbf{O}_{p}\left(G_{\alpha}\right) \cap N_{\alpha \beta}=$ 1 , there exists a prime $t \neq p$ which divides $\left|N_{\alpha \beta}\right|$. This prime $t$ divides $\left|T_{i \delta_{i}}\right|$, and it follows that $N_{\alpha}$ contains a subgroup which is isomorphic to $\mathbb{Z}_{t}^{k}$. A Sylow $t$-subgroup of $N_{\alpha}$ is isomorphic to a Sylow $t$-subgroup of $N_{\alpha} / \mathbf{O}_{p}\left(G_{\alpha}\right)$, which is isomorphic to a subgroup of $G_{\alpha \beta}=\left(G_{\alpha}^{[1]} \times G_{\beta}^{[1]}\right)$. $O$. Since both $G_{\alpha}^{[1]}$ and $G_{\alpha \beta} / G_{\alpha}^{[1]}$ are subgroups of $\Gamma \mathrm{L}_{1}\left(p^{e}\right)$ which are metacyclic, it follows that $k \leq 4$, and so $k=3$.

Let $L$ be the kernel of $G^{+}$acting on $\left\{T_{1}, T_{2}, T_{3}\right\}$ by conjugation. Then $T_{1} \times T_{2} \times$ $T_{3}=N \triangleleft L \leq \tilde{T}_{1} \times \tilde{T}_{2} \times \tilde{T}_{3}$, where $T_{i} \leq \tilde{T}_{i} \leq \operatorname{Aut}\left(T_{i}\right)$ such that $\tilde{T}_{i} \cong L / \mathbf{C}_{L}\left(T_{i}\right)$. Since $T_{1}, T_{2}, T_{3}$ are conjugate in $G^{+}$, we have $\tilde{T}_{1} \cong \tilde{T}_{2} \cong \tilde{T}_{3}$. Each element $g \in L$ can be written as

$$
g=\left(t_{1}, t_{2}, t_{3}\right), \quad \text { for some } t_{i} \in \tilde{T}_{i} .
$$

Let $\pi_{i}$ be the projection from $L$ to the $i$ th coordinate, namely $\pi_{i}(g)=t_{i}$. Then $\pi_{i}(L) \cong \tilde{T}_{i}$.

Now $X:=\left(\tilde{T}_{1} \times \tilde{T}_{2} \times \tilde{T}_{3}\right) \leq \operatorname{Sym}(\Delta) 2 S_{3}$, and hence for a point $\alpha=\left(\delta_{1}, \delta_{2}, \delta_{3}\right) \in$ $\Omega$, the stabilizer $X_{\alpha}$ is $\tilde{T}_{1 \delta_{1}} \times \tilde{T}_{2 \delta_{2}} \times \tilde{T}_{3 \delta_{3}}$. Since $\pi_{i}(L)=\tilde{T}_{i}$, we have $\pi_{i}\left(L_{\alpha}\right)=\tilde{T}_{i \delta_{i}}$. By Lemma 3.2, $p^{e}-1$ has a primitive prime divisor $r \geq 5$. Since $\left(p^{e}-1\right) / 2$ divides $\left|G_{\alpha}\right|$, so does $r$. Further, as $G_{\alpha}^{+} / L_{\alpha} \cong G^{+} / L \leq \mathrm{S}_{3}$, we conclude that $r|| L_{\alpha} \mid$, and so $r$ divides $\left|\tilde{T}_{i \delta_{i}}\right|$.

By Theorem 3.1, $\mathbb{Z}_{p}^{e} \cong \mathbf{O}_{p}\left(G_{\alpha}\right) \triangleleft N_{\alpha} \triangleleft G_{\alpha}$, and hence

$$
\mathbb{Z}_{p}^{l} \cong \pi_{i}\left(\mathbf{O}_{p}\left(G_{\alpha}\right)\right) \triangleleft \pi_{i}\left(N_{\alpha}\right) \cong T_{i \delta_{i}} \triangleleft \tilde{T}_{i \delta_{i}},
$$

where $l=e / 3$. We may write $\tilde{T}_{i \delta_{i}}=\mathbb{Z}_{p}^{l}: K_{i}$. Then $r$ divides $\left|K_{i}\right|$.

Let $C_{i}$ be a Sylow $r$-subgroup of $K_{i}$, and consider the subgroup $M_{i}$ : = $\pi_{i}\left(\mathbf{O}_{p}\left(G_{\alpha}\right)\right): C_{i}$ of $\tilde{T}_{i}$. If $C_{i}$ acts non-trivially on $\pi_{i}\left(\mathbf{O}_{p}\left(G_{\alpha}\right)\right) \cong \mathbb{Z}_{p}^{l}$, then $C_{i}<$ $\mathrm{GL}_{l}(p)$, which contradicts the fact that $r \nmid p^{l}-1$. Thus $M_{i}=\pi_{i}\left(\mathbf{O}_{p}\left(G_{\alpha}\right)\right) \times C_{i}$. Notice that a Sylow $r$-subgroup of $G_{\alpha}$ is contained in a Sylow $r$-subgroup of $\tilde{T}_{1} \times \tilde{T}_{2} \times \tilde{T}_{3}$, it follows that all the $r$-elements of $G_{\alpha}$ are in the centralizer of $\mathbf{O}_{p}\left(G_{\alpha}\right)$, which is $G_{\alpha}^{[1]}$ by Theorem 3.1. Since $r$ does not divide $\left|G_{\alpha}^{\Gamma(\alpha)}\right|$, we again obtain a contradiction.

Hence $G^{+}$is not of product action type, that is, the primitive type of $G^{+}$is affine or almost simple.

\section{Proofs of the main theorems}

The remaining part of this paper is devoted to complete the proofs of our main theorems. The proofs of the Theorem 1.1 and Theorem 1.2 depend on the classification, 
by Liebeck and Saxl [14], of primitive permutation groups of which the point stabilizer has odd order.

Theorem 4.1 [14, Theorem 2] Let $G$ be an almost simple group with $T=\operatorname{soc}(G)$. Assume that $M$ is a maximal subgroup of $G$ of odd order. Then one of the following holds:

(i) $G=\mathrm{A}_{p}$, and $M=\mathbb{Z}_{p}: \mathbb{Z}_{(p-1) / 2}$, where $p \equiv 3(\bmod 4)$ is a prime, and $p \neq 7,11$ or 23;

(ii) $T=\operatorname{PSL}_{2}\left(p^{e}\right)$, and $T \cap M=\mathbb{Z}_{p}^{e}: \mathbb{Z}_{\left(p^{e}-1\right) / 2}$, where $p$ is a prime, and $p^{e} \equiv$ $3(\bmod 4)$;

(iii) $T=\operatorname{PSL}_{r}(q)$, and $T \cap M=\mathbb{Z}_{\left(q^{r}-1\right) /(q-1)(r, q-1)}: \mathbb{Z}_{r}$, where $r$ is an odd prime;

(iv) $G=\operatorname{PSL}_{3}$ (4).3, and $M=\left(\mathbb{Z}_{7}: \mathbb{Z}_{3}\right) \times \mathbb{Z}_{3}$;

(v) $T=\operatorname{PSU}_{r}(q)$, and $T \cap M=\mathbb{Z}_{\left(q^{r}+1\right) /(q+1)(r, q+1)}: \mathbb{Z}_{r}$, where $r$ is an odd prime, and $(r, q) \neq(3,3),(5,2)$ or $(3,5)$;

(vi) $G=\operatorname{PSU}_{3}(5) \cdot 3$, and $M=\left(\mathbb{Z}_{7}: \mathbb{Z}_{3}\right) \times \mathbb{Z}_{3}$.

(vii) $G$ and $M$ lie in the following table:

\begin{tabular}{lllll}
\hline$G$ & $\mathrm{M}_{23}$ & Th & B & M \\
\hline$M$ & $\mathbb{Z}_{23}: \mathbb{Z}_{11}$ & $\mathbb{Z}_{31}: \mathbb{Z}_{15}$ & $\mathbb{Z}_{47}: \mathbb{Z}_{23}$ & $\mathbb{Z}_{59}: \mathbb{Z}_{29}, \mathbb{Z}_{71}: \mathbb{Z}_{35}$ \\
\hline
\end{tabular}

As shown in the examples of the last section, almost all of the pairs $(G, M)$ in Theorem 4.1 do give rise to $(G, 2)$-path-transitive graphs which are $G$-vertex primitive or $G$-vertex biprimitive.

\subsection{Proof of Theorem 1.1}

Let $\Gamma$ be a $G$-vertex-primitive and $(G, 2)$-path-transitive but $(G, 2)$-arc-intransitive graph, of valency $k$. Then $G_{\alpha}$ is primitive on $\Gamma(\alpha)$, and by Theorem $3.1, k=p^{e} \equiv$ $3(\bmod 4)$ with $p$ prime. By Lemma 3.4, the primitive type of $G$ is affine or almost simple. Assume that $G$ is of affine type, with $N:=\operatorname{soc}(G)=\mathbb{Z}_{p}^{n}$. Then we may identify $V \Gamma$ with $N$ such that $\alpha$ is the zero vector. Let $\beta \in \Gamma(\alpha)$. Then the pair $\{\beta,-\beta\}$ is a block of imprimitivity in $\Gamma(\alpha)$ of $G_{\alpha}$. Since $G_{\alpha}$ is 2-homogeneous on $G_{\alpha}$, we have $\beta=-\beta$, so $p=2$, as in (1) of Theorem 1.1. Assume that $G$ is almost simple, and $T \leq G \leq \operatorname{Aut}(T)$, with $T$ a non-abelian simple group. Then the pair $\left(G, G_{\alpha}\right)$ appears in Theorem 4.1. Thus we need to analyse the pairs of Theorem 4.1 in turn.

(1) Assume that $\left(G, G_{\alpha}\right)=\left(\mathrm{A}_{p}, \mathbb{Z}_{p}: \mathbb{Z}_{(p-1) / 2}\right),\left(\mathrm{Th}, \mathbb{Z}_{31}: \mathbb{Z}_{15}\right),\left(\mathrm{B}, \mathbb{Z}_{47}: \mathbb{Z}_{23}\right)$, $\left(\mathrm{M}, \mathbb{Z}_{59}: \mathbb{Z}_{29}\right)$, or $\left(\mathrm{M}, \mathbb{Z}_{71}: \mathbb{Z}_{35}\right)$, or $\left(T, T_{\alpha}\right)=\left(\mathrm{PSL}_{2}\left(p^{e}\right), \mathbb{Z}_{p}^{e}: \mathbb{Z}_{\left(p^{e}-1\right) / 2}\right)$. Then by Examples 2.2, 2.4, and 2.9, each of these cases corresponds to a $G$-vertex-primitive, $(G, 2)$-path-transitive but not $(G, 2)$-arc-transitive graph, as in Theorem 1.1.

(2) Assume that $\left(G, G_{\alpha}\right)=\left(\mathrm{M}_{23}, \mathbb{Z}_{23}: \mathbb{Z}_{11}\right)$. We write $G_{\alpha}$ as $K: L$, where $K \cong \mathbb{Z}_{23}, L \cong \mathbb{Z}_{11}$. Then $G_{\alpha}$ is the normalizer of a Sylow-23 subgroup of $\mathrm{M}_{23}$. By the Atlas [5], the cyclic subgroups of order 11 form two conjugacy classes in $G$. For $L \cong \mathbb{Z}_{11}$, assume that the order $\left|\mathbf{N}_{G}(L)\right|$ is even. Then $\mathbf{N}_{G}(L)$ is contained in a maximal subgroup isomorphic to $\mathrm{M}_{11}$ or $\mathrm{M}_{22}$. Suppose that $\mathbf{N}_{G}(L)<M$, where 
$M$ is $\mathrm{M}_{11}$ or $\mathrm{M}_{22}$. Then it follows that $\mathbf{N}_{G}(L)=\mathbf{N}_{M}(L)$, and by the Atlas [5], $\mathbf{N}_{M}(L)=\mathbb{Z}_{11}: \mathbb{Z}_{5}$, a contradiction. Thus there is no $(G, 2)$-path-transitive but $(G, 2)$ arc-intransitive graph occurring in this case.

(3) Assume that $\left(T, T_{\alpha}\right)=\left(\operatorname{PSL}_{r}(q), \mathbb{Z}_{\left(q^{r}-1\right) /(q-1)(r, q-1)}: \mathbb{Z}_{r}\right)$. Define $m=\left(q^{r}-\right.$ 1) $/(q-1)(r, q-1)$. Then

$$
G_{\alpha}=\left(\mathbb{Z}_{m}: \mathbb{Z}_{r}\right) . o
$$

where $o=G / T$. By Theorem 3.1, the unique minimal normal subgroup of $G_{\alpha}$ is an elementary abelian group, so $m$ is a prime, and $r=(m-1) / 2$. Notice that if $q=p^{e}$ with $p \geq 3$, then $r<(m-1) / 2$. Thus $p=2$ and $(r, q)=(3,4)$, and $\left(G, G_{\alpha}\right)=$ $\left(\mathrm{PGL}_{3}(4), 7: 3 \times 3\right)$. If there exists a 2-path-transitive and 2-arc-intransitive graph $\Gamma$, let $(\alpha, \beta)$ be an arc of $\Gamma$. Then $\Gamma=\operatorname{Cos}\left(G, G_{\alpha}, G_{\alpha} g G_{\alpha}\right)$, with the 2-element $g$ interchanging $\alpha$ and $\beta$. Assume that $G_{\alpha}=(\langle x\rangle:\langle y\rangle) \times\langle z\rangle$, where $o(x)=7, o(y)=$ $o(z)=3$, and $z$ is a diagonal automorphism. Then $G_{\alpha}^{[1]}=\langle z\rangle, G_{\beta}^{[1]}=\langle y\rangle$, and $g$ interchanges $G_{\alpha}^{[1]}$ with $G_{\beta}^{[1]}$. Checking the Atlas [5], $L:=\langle y\rangle \times\langle z\rangle$ is contained in a subgroup isomorphic to $\mathbb{Z}_{3}^{2}: 2 \mathrm{~A}_{4}$. Thus $y \in \mathbb{Z}_{3}^{2}$, and $z \in 2 \mathrm{~A}_{4}$, but it is easy to see that no 2-element of $2 \mathrm{~A}_{4}$ interchanges $\langle y\rangle$ with $\langle z\rangle$, a contradiction (actually by GAP [9], $\left.\mathbf{N}_{G}(L)=27\right)$. Thus in this case there exists no $(G, 2)$-path-transitive but $(G, 2)$-arcintransitive graph.

(4) Assume that $\left(T, T_{\alpha}\right)=\left(\operatorname{PSU}_{r}(q), \mathbb{Z}_{\left(q^{r}+1\right) /(q+1)(r, q+1)}: \mathbb{Z}_{r}\right)$. Then by a similar argument to the one above, the only possibility is $\left(G, G_{\alpha}\right)=\left(\operatorname{PGU}_{3}(5), 7: 3 \times 3\right)$. Denote by $(\langle x\rangle:\langle y\rangle) \times\langle z\rangle$ the subgroup 7:3 $\times 3$, where $o(x)=7, o(y)=o(z)=3$, and let $L=\langle y\rangle \times\langle z\rangle$. Using the "pq-package" of GAP [2], then calculation shows that $\left|\mathbf{N}_{G}(L)\right|=27$. Thus there exists no 2-element $g$ which satisfies the conditions of Lemma 2.1, so no such graph exists.

This completes the proof of Theorem 1.1.

Let $\Gamma$ be a (directed or undirected) graph with vertex set $V$. Then the standard double cover of $\Gamma$ is defined to be the undirected bipartite graph $\tilde{\Gamma}$ with parts $V_{0}$ and $V_{1}$, where $V_{i}=\{(v, i) \mid v \in V\}$, such that two vertices $(x, i)$ and $(y, j)$ are adjacent if and only if $i \neq j$, and $x, y$ are adjacent in $\Gamma$.

Lemma 4.2 Let $\Gamma=\operatorname{Cos}(G, H, H g H)$ be a $G$-vertex-primitive, and $(G, 2)$-pathtransitive but $(G, 2)$-arc-intransitive graph. Let $\Sigma=\operatorname{Cos}\left(K, H_{1}, H_{1}(g, z) H_{1}\right)$, where $K=G \times\langle z\rangle, H_{1}=H \times\{1\}$, and $z$ is an involution. Then

(1) $\Sigma$ is isomorphic to the standard double cover of $\Gamma$.

(2) $\Sigma$ is $K$-vertex-biprimitive, $(K, 2)$-path-transitive and $(K, 2)$-arc-intransitive.

Proof (1) We have $V \Gamma=[G: H]=\{H x \mid x \in G\}$. Let $\tilde{\Gamma}$ denote the standard double cover of $\Gamma$. Then $V \tilde{\Gamma}=\{(H x, i) \mid x \in G, i=0$ or 1$\}$. Define a map $\psi: V \tilde{\Gamma} \mapsto V \Sigma$ as follows:

$$
\psi((H x, i))=H_{1}\left(x, z^{i}\right)
$$


This map is clearly one-to-one. Notice that $z^{-i}=z^{i}$, for $x, y \in G$ and $i, j \in\{0,1\}$, we have

$$
\begin{aligned}
\{(H x, i),(H y, j)\} \in E \tilde{\Gamma} & \Longleftrightarrow y x^{-1} \in H g H, \quad \text { and } \quad i+j=1 \\
& \Longleftrightarrow\left(y x^{-1}, z^{i+j}\right) \in H_{1}(g, z) H_{1} \\
& \Longleftrightarrow\left(y, z^{j}\right)\left(x, z^{i}\right)^{-1} \in H_{1}(g, z) H_{1} \\
& \Longleftrightarrow\left\{H_{1}\left(x, z^{i}\right), H_{1}\left(y, z^{j}\right)\right\} \in E \Sigma .
\end{aligned}
$$

Therefore $\tilde{\Gamma} \cong \Sigma$.

(2) Let $\alpha=H \in V \Gamma$, and let $\alpha_{1}=H_{1} \in V \Sigma$. Then it is easy to show that $G_{\alpha}^{\Gamma(\alpha)}$ is permutationally isomorphic to $K_{\alpha_{1}}^{\Sigma\left(\alpha_{1}\right)}$. It follows that $\Sigma$ is $K$-vertex-biprimitive, and $(K, 2)$-path-transitive but not $(K, 2)$-arc-transitive.

\subsection{Proof of Theorem 1.2}

Let $\Gamma$ be a $G$-vertex-biprimitive, $(G, 2)$-path-transitive but $(G, 2)$-arc-intransitive graph, with two parts $\Delta$ and $\Delta^{\prime}$. Then by Lemma 3.4, the primitive type of $G_{\Delta}$ is affine, or almost simple. If $\mathbf{C}_{G}\left(G_{\Delta}\right)$ is non-trivial, then since $\left|G: G_{\Delta}\right|=2$, we have $G=G_{\Delta} \times \mathbb{Z}_{2}$. Therefore $\Gamma$ is the standard double cover of one of the graphs in Theorem 1.1. Combining this with Lemma 4.2, we come to the conclusion (1) of Theorem 1.2. Thus we assume that $\mathbf{C}_{G}\left(G_{\Delta}\right)=1$.

Assume first that $G_{\Delta}$ is of affine type. We consider two cases: $G_{\Delta}$ is faithful on $\Delta$, and $G_{\Delta}$ is unfaithful on $\Delta$.

Suppose that $G_{\Delta}$ is faithful on $\Delta$. Since $G_{\Delta}$ is not regular on $\Delta$, and $\mathbf{C}_{G}\left(G_{\Delta}\right)=1$, we have $\operatorname{soc}(G)=\operatorname{soc}\left(G_{\Delta}\right) \cong \mathbb{Z}_{r}^{d}$ with $r$ prime. Therefore for $\alpha \in \Delta$, there exist a prime number $p$ and an odd number $e$, such that $p^{e} \equiv 3(\bmod 4)$, and $G_{\alpha}=\left(G_{\Delta}\right)_{\alpha}<$ $\mathrm{A} \Gamma \mathrm{L}_{1}\left(p^{e}\right)$ is 2-homogeneous but not 2-transitive of degree $p^{e}$, and is an irreducible subgroup of $\mathrm{GL}_{d}(r)$, as in (2) of Theorem 1.2.

Suppose that $G_{\Delta}$ is unfaithful on $\Delta$. Since $\Gamma$ is $G$-vertex-biprimitive, the kernel $G_{(\Delta)}$ is transitive on $\Delta^{\prime}$, it follows that $\Gamma=\mathrm{K}_{p^{e}}, p^{e}$, where $p^{e}=|\Delta|=\left|\Delta^{\prime}\right|$, with $p$ prime, $e$ odd. For $\alpha \in \Delta$, we have $\Gamma(\alpha)=\Delta^{\prime}$, and $G_{\alpha}^{\Delta^{\prime}}$ is 2-homogeneous but not 2transitive, so $G_{\alpha}^{\Delta^{\prime}}=\mathbb{Z}_{p}^{e}: G_{\alpha \beta}$. By Theorem 3.1, we have $G_{\alpha \beta} \leq \Gamma \mathrm{L}_{1}\left(p^{e}\right) \times \Gamma \mathrm{L}_{1}\left(p^{e}\right)$. It follows that $G=\left(\mathbb{Z}_{p}^{e} \times \mathbb{Z}_{p}^{e}\right): G_{\alpha \beta}: \mathbb{Z}_{2}$, as in (3) of Theorem 1.2.

Assume next that $G_{\Delta}$ is almost simple. Then the pair $\left(G_{\Delta},\left(G_{\Delta}\right)_{\alpha}\right)=\left(G_{\Delta}, G_{\alpha}\right)$ is given in Theorem 4.1. Thus we need to consider all the candidates in Theorem 4.1.

(a) Assume that $\operatorname{soc}\left(G_{\Delta}\right)=\mathrm{A}_{p}$ with $p \geq 5$. Then $\left(G, G_{\alpha}\right)=\left(\mathrm{S}_{p}, \mathbb{Z}_{p}: \mathbb{Z}_{(p-1) / 2}\right)$, with $p$ prime, $p \equiv 3(\bmod 4)$ and $p \neq 7,11,23$. By Example 2.4 and Lemma 2.5 , there exists a graph $\Sigma=\operatorname{Cos}\left(G, G_{\alpha}, G_{\alpha} f G_{\alpha}\right)$, which is $G$-vertex-biprimitive, $(G, 2)$-path-transitive but not $(G, 2)$-arc-transitive, with valency $k=p$. Further, Aut $\Sigma=\mathrm{S}_{p}$, as indicated in Theorem 1.2.

(b) Assume that $\operatorname{soc}\left(G_{\Delta}\right)$ is a sporadic almost simple group. Then by Theorem $4.1, \operatorname{soc}\left(G_{\Delta}\right)=\mathrm{M}_{23}$, Th, B or $\mathrm{M}$. For each case, since $\mathbf{C}_{G}\left(G_{\Delta}\right)=1$, and $\operatorname{Out}\left(\operatorname{soc}\left(G_{\Delta}\right)\right)=1$, we obtain a contradiction. Thus there is no $G$-vertex-biprimitive, and $(G, 2)$-path-transitive but $(G, 2)$-arc-intransitive graph arising in this case. 
(c) Assume that $\operatorname{soc}\left(G_{\Delta}\right)$ is a classical group of Lie type. Then by Theorem 4.1, we need to consider the following cases:

either $\left(\operatorname{soc}\left(G_{\Delta}\right), \operatorname{soc}\left(G_{\Delta}\right)_{\alpha}\right)=\left(\operatorname{PSL}_{2}(q), \mathbb{Z}_{p}^{e}: \mathbb{Z}_{\left(p^{e}-1\right) / 2}\right)$, where $q=p^{e} \equiv$ $3(\bmod 4)$, or $\left(G, G_{\alpha}\right)=\left(\mathrm{PGL}_{3}(4) .\langle\tau\rangle, 7: 3 \times 3\right)$ or $\left(\mathrm{PGU}_{3}(5) .\langle\tau\rangle, 7: 3 \times 3\right)$, where $\tau$ is an involution.

Assume that $\left(\operatorname{soc}\left(G_{\Delta}\right), \operatorname{soc}\left(G_{\Delta}\right)_{\alpha}\right)=\left(\operatorname{PSL}_{2}(q), \mathbb{Z}_{p}^{e}: \mathbb{Z}_{\left(p^{e}-1\right) / 2}\right)$. Then $G=$ $\operatorname{PSL}_{2}(q) .2$ and $G_{\alpha}=\operatorname{soc}\left(G_{\Delta}\right)_{\alpha}=\mathbb{Z}_{p}^{e}: \mathbb{Z}_{\left(p^{e}-1\right) / 2}=K: L$, say. Let $T:=\operatorname{PSL}_{2}(q)$. Then by a result of Dickson in 1901 (see [6, p. 263]), $\mathbf{N}_{G}(K) \cong \mathbb{Z}_{p}^{e}: \mathbb{Z}_{\left(p^{e}-1\right)}$, $\mathbf{N}_{G}(L) \cong \mathrm{D}_{2(q-1)}$, and $\mathbf{N}_{T}(L) \cong \mathrm{D}_{(q-1)}$. Write $\mathbf{N}_{G}(L)=\langle x\rangle:\langle\delta\rangle$, where $o(x)=$ $q-1$, and $o(\delta)=2$. Let $g:=x^{\frac{q-1}{2}} \delta$. Since $\delta \in T$ and $\delta$ does not normalize $K$, we have $g \in G \backslash T$ is a 2-element, and $g \in \mathbf{N}_{G}(L) \backslash \mathbf{N}_{G}(K)$. Thus the graph $\Gamma=\operatorname{Cos}\left(G, G_{\alpha}, G_{\alpha} g G_{\alpha}\right)$ is $G$-vertex-biprimitive, $(G, 2)$-path-transitive but $(G, 2)$ arc-intransitive. Since the order of $\Gamma$ is $2(q+1)$, and the valency of $\Gamma$ is $q$, we conclude that $\Gamma=\mathrm{K}_{q+1, q+1}-(q+1) \mathrm{K}_{2}$. Thus $\Gamma$ is the standard double cover of the complete graph $\mathrm{K}_{q+1}$, and therefore (1) holds.

Assume that $\left(G, G_{\alpha}\right)=\left(\mathrm{PGL}_{3}(4) \cdot\langle\tau\rangle, 7: 3 \times 3\right)$. Then by Example 2.11, there exists a graph $\Gamma=\operatorname{Cos}\left(G, G_{\alpha}, G_{\alpha} g G_{\alpha}\right)$ which is $G$-vertex-biprimitive, $(G, 2)$-pathtransitive but $(G, 2)$-arc-intransitive. Further, by Lemma 2.13, Aut $\Gamma=G .2$, as shown in (5) of Theorem 1.2.

Assume that $\left(G, G_{\alpha}\right)=\left(\mathrm{PGU}_{3}(5) \cdot\langle\tau\rangle, 7: 3 \times 3\right)$. Then by Example 2.12 , there exists a graph $\Gamma=\operatorname{Cos}\left(G, G_{\alpha}, G_{\alpha} g G_{\alpha}\right)$ which is $G$-vertex-biprimitive, $(G, 2)$-pathtransitive but $(G, 2)$-arc-intransitive, with Aut $\Gamma=G$, as shown in (6) of Theorem 1.2.

This completes the proof of Theorem 1.2.

Recall that the line-graph $\mathbf{L}(\Gamma)$ of a graph $\Gamma=(V, E)$ is the graph with vertex set $E$ such that two vertices in $\mathbf{L}(\Gamma)$ are adjacent if and only if they are incident in $\Gamma$. It was shown in [12] that a graph is 2-path-transitive but 2-arc-intransitive if and only if its line-graph is half-arc-transitive.

\subsection{Proof of Theorem 1.3}

Let $G$ be a group given in the first column of Table 1. Then by Theorems 1.1 and 1.2, there exists a connected $(G, 2)$-path-transitive graph $\Gamma$ such that Aut $\Gamma=G$, the vertex-stabilizer $G_{\alpha}$, and the valency $k$ lie in the following table, where $p \equiv 3(\bmod 4)$ and $p \neq 7,11$ or 23 :

\begin{tabular}{|c|c|c|c|c|c|c|}
\hline$G$ & $\mathrm{P} \Gamma \mathrm{U}_{3}(5)$ & Th & B & M & $\mathrm{A}_{p}$ & $\mathrm{~S}_{p}$ \\
\hline & $\times \mathbb{Z}_{3}$ & $\mathbb{Z}_{31}: \mathbb{Z}_{15}$ & $\mathbb{Z}_{23}$ & $z_{29}$, & $(p-1) / 2$ & $\mathbb{Z}_{p}:$ \\
\hline$k$ & 7 & 31 & 47 & 59,71 & $p$ & $p$ \\
\hline
\end{tabular}

Let $\Sigma$ be the line graph of $\Gamma$. Then the valency of $\Sigma$ equals $2(k-1)$, which is the value of $m$ given in the third column of Table 1. By [12, Theorem 1.3], a graph is 2-path-transitive but not 2-arc-transitive if and only if its line graph is halfarc-transitive. Since the graph $\Gamma$ has more than five vertices, a result of Whitney (1932) (see [1]) implies that Aut $\Sigma \cong$ Aut $\Gamma=G$. It follows that the line graph $\Sigma$ 
is half-arc-transitive. An edge $\{\alpha, \beta\}$ of $\Gamma$ is a vertex of $\Sigma$, denoted by $v$. Thus the vertex-stabilizer $G_{v}$ for $\Sigma$ is the stabilizer of the edge $\{\alpha, \beta\}$. In particular, the vertexstabilizer $G_{v}$ is the subgroup $H$ listed in the second column of Table 1.

Acknowledgements This work forms a part of the second author's Ph.D. project, and is partially supported by an ARC Discovery Project Grant.

The authors would like to express their heartfelt thanks to the referees for the helpful comments and suggestions which made this paper substantially improved.

\section{References}

1. Babai, L.: Automorphism Groups, Isomorphism, Reconstruction. Handbook of Combinatorics, vol. 2, pp. 1447-1540. MIT Press, Cambridge (1995)

2. Beule, J.D., Govaerts, P., Hallez, A., Lemens, P.: Projective Geometries package for GAP. URL http://cage.ugent.be/jdebeule/pg.html

3. Biggs, N.: Algebraic Graph Theory, 2nd edn. Cambridge University Press, New York (1992)

4. Conder, M.D.E., Praeger, C.E.: Remarks on path-transitivity in finite graphs. Eur. J. Comb. 17, 371378 (1996)

5. Conway, J.H., Curtis, R.T., Norton, S.P., Parker, R.A., Wilson, R.A.: Atlas of Finite Groups. Oxford University Press, London/New York (1985)

6. Dickson, L.E.: Linear Groups: With an Exposition of the Galois Field Theory. Dover, New York (1958)

7. Dixon, J.D., Mortimer, B.: Permutation Groups. Springer, New York (1996)

8. Fang, X.G., Li, C.H., Wang, J.: Finite vertex primitive 2-arc regular graphs. J. Algebr. Comb. 25, 125-140 (2007)

9. GAP: Groups, algorithms, and programming—a system for computational discrete algebra, version 4.4.12. URL http://www.gap-system.org

10. Huppert, B.: Finite Groups. Springer, Berlin (1968)

11. Ivanov, A.A., Praeger, C.E.: On finite affine 2-arc-transitive graphs. Eur. J. Comb. 14, 421-444 (1993)

12. Li, C.H., Zhang, H.: On finite 2-path-transitive graphs. Submitted

13. Li, C.H., Niu, L., Seress, A., Solomon, R.: The vertex-primitive and biprimitive $s$-arc-regular graphs. J. Comb. Theory, Ser. B 100, 359-366 (2010)

14. Liebeck, M., Saxl, J.: On point stabilizers in primitive permutation groups. Commun. Algebra 19, 2777-2786 (1991)

15. Liebeck, M., Praeger, C.E., Saxl, J.: The maximal factorisations of the finite simple groups and their automorphism groups. Mem. Am. Math. Soc. 86, 432 (1990)

16. Marušič, D., Xu, M.Y.: A 1/2-transitive graph of valency 4 with a nonsolvable group of automorphisms. J. Graph Theory 25, 133-138 (1997)

17. Praeger, C.E.: An O'Nan-Scott theorem for finite quasiprimitive permutation groups and an application to 2-arc transitive graphs. J. London Math. Soc. Ser. 24 7, 227-239 (1993)

18. Weiss, R.M.: $s$-arc transitive graphs. In: Algebraic Methods in Graph Theory, vol. 2, pp. 827-847 (1981) 